\title{
Design of optoelectronic system for optical diffusion tomography
}

\author{
Igor Erakhtin ${ }^{1,}$, Aleksandr Aristov ${ }^{1}$, Anna Novoseltseva $^{2}$, and Viktor Sukhanov ${ }^{3}$ \\ ${ }^{1}$ Tomsk Polytechnic University, 634050 Tomsk, Russia \\ ${ }^{2}$ Worcester Polytechnic Institute, MA 01609-2280 Worcester, USA \\ ${ }^{3}$ Siberian Branch, Russian Academy of Sciences, 630090, Novosibirsk, Russia
}

\begin{abstract}
This article explores issues connected with the circuit design of a device for optical diffusion tomography, which we are currently designing. We plan to use the device in experimental studies for the development of a faster method of brain hematoma detection. We reviewed currently existing methods for emergency diagnosis of hematomas, primarily the Infrascanner model 2000, for which we identified weaknesses, and outlined suggestions for improvements. This article describes the method of scanning tissues based on a triangulated arrangement of sources and receivers of optical radiation, and it discusses the optoelectronic system that implements that principle.
\end{abstract}

\section{Introduction}

Brain injuries are often accompanied by intracranial hematomas, the presence of which is life-threatening. Therefore, quick detection of hematoma, including its size and location, is important for effective medical care. Today, there are two primary methods for the detection of intracranial hematomas: magnetic resonance and computed tomography. Carrying out an examination of patients by these methods is not always possible, since they are not always accessible. Therefore, creating a portable device that is able to identify the presence of intracranial injuries is important. A portable device can be achieved using spectroscopy in the optical range of wavelengths.

\section{Problem definition}

Nowadays, there is a device on the market, Infrascanner model 2000 [1] developed by InfraScan company. It is a portable detector of intracranial hematomas, operating in the near infrared range $(808 \mathrm{~nm})$. According to the experimental investigations [2, 3], the Infrascanner model 2000 has a high diagnostic sensitivity. The company published that the probability of detecting the presence of hematomas is $91 \%$ (assuming that the occurrence of hematoma is not more than $2.5 \mathrm{~cm}$ from the brain cortex and is not larger than $3.5 \mathrm{~cm}^{3}$ ). Also, the device does not determine the parameters of the hematoma (size, depth of bedding, exact localization) which are very important for resuscitation and rehabilitation.

* Corresponding author: igor_e_k_94@mail.ru 
The operation principle of the Infrascanner model 2000 is based on a differential method, i.e. the identification of hematomas is done by comparing the optical properties of symmetrical sections on the head at the given points [1]. Thus, there is a high probability of missing small or deeply bedded hematomas outside of these zones. In order to eliminate these disadvantages, improvements of the method and the device which will help in collecting qualitative and quantitative data (e.g. hematoma parameters) are necessary.

\section{Results}

The Infrascanner devices use only one radiation source and a receiver, which limits its diagnostic capabilities. An increase of the number of sources and photodetectors may help to improve hematoma parameters detection. We assume that the optimal scheme is a scheme with radiation sources and detectors located at the vertices of an equilateral triangle (Figure 1).

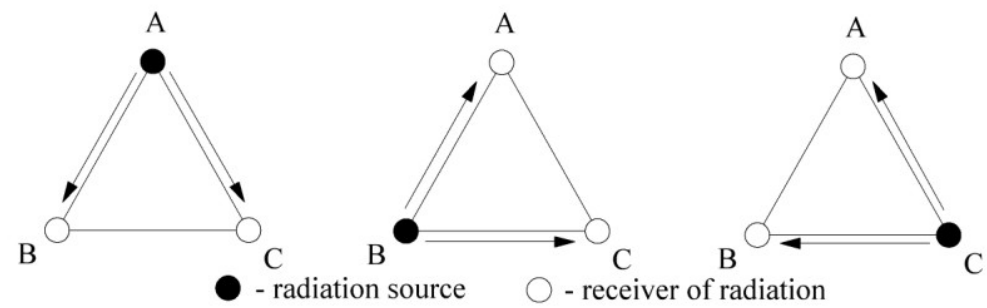

Fig. 1. Scheme of triangulation method.

As shown in Figure 1, the radiation source is located at point $\mathrm{A}$ and the receivers at points $\mathrm{C}$ and $\mathrm{B}$, respectively, this configuration is used for the first measurement. Then the source moves to point $\mathrm{C}$, and the receivers are located at points $\mathrm{A}$ and $\mathrm{B}$ during the second measurement. Finally, the source moves to point B, and the receivers are at points $\mathrm{A}$ and $\mathrm{C}$ during the third measurement. As a result of these measurements, we can detect shape and location of hematoma.

Additionally, two-wave spectroscopy can be used to increase the examination sensitivity. A hematoma is a clot of coagulated blood. The earlier works [4] have shown that there is a significant spectral difference for clotted blood and incoagulated blood in the red region of the spectrum. Therefore, the measurement can be carried out in the red (about $640 \mathrm{~nm}$ ) and infrared (about $805 \mathrm{~nm}$ ) regions of the spectrum. A number of issues relating to the development of such systems for hematoma detection were considered in publications $[5,6]$.

In view of the device requirements and research objectives, it was proposed to construct an experimental model, as described below.

The system sequentially reads the information from two photodetectors that collect a red and an infrared radiation transmitted through the sample. Therefore, the device consistently generates two pulses of red and infrared light with a minimum time delay. Then, these two radiations that passed through the examined biological tissue are measured and the results are saved. Further, a cycle of measurements is carried out for the subsequent combinations of radiation sources and receivers. Then the information is analyzed and the results are displayed on an indicatory device. On the basis of the abovementioned, one of the best solutions is a creation of the device based on a microcontroller, that generates pulse sequences to measure parameters of the signal, data analysis and its display on the indicatory device.

We use laser diodes as the radiation sources since they have high-speed performance 
and sufficient emissive power. We use a voltage-to-current converter as a supply source for an adjustment of the laser diode current. For the photo-sensor element, it is better to use photodiodes since in comparison with other similar photo-sensor elements the photodiode has high integral sensitivity, a wide range of wavelengths, the linear dependence of a photoelectric current of the diode from emissive power, as a result - simpler data analysis. As the signal from the photodiode is very small, it needs to be amplified to an appropriate level. In addition, an ambient illumination interferes the signal on the photodiodes. In order to eliminate this interference, the method of measuring the backlight voltage and subtracting it before supplying the laser diodes can be used. Thus, it will help to get rid of a constant component of backlight voltage, and digital filtering will be used for elimination of $50 \mathrm{~Hz}$ and $100 \mathrm{~Hz}$ signals. After filtering, the signal will be amplified to the level of analog-to-digital converter (ADC) operation. Since head tissues in different regions of a head have significant variance of the optical properties, the final amplifier needs an automatic gain control. For the realization of the triangulation method, there are should be three receiving and transmitting channels. Schematic diagram of our optoelectronic system (which includes abovementioned modifications) is shown in Figure 2.

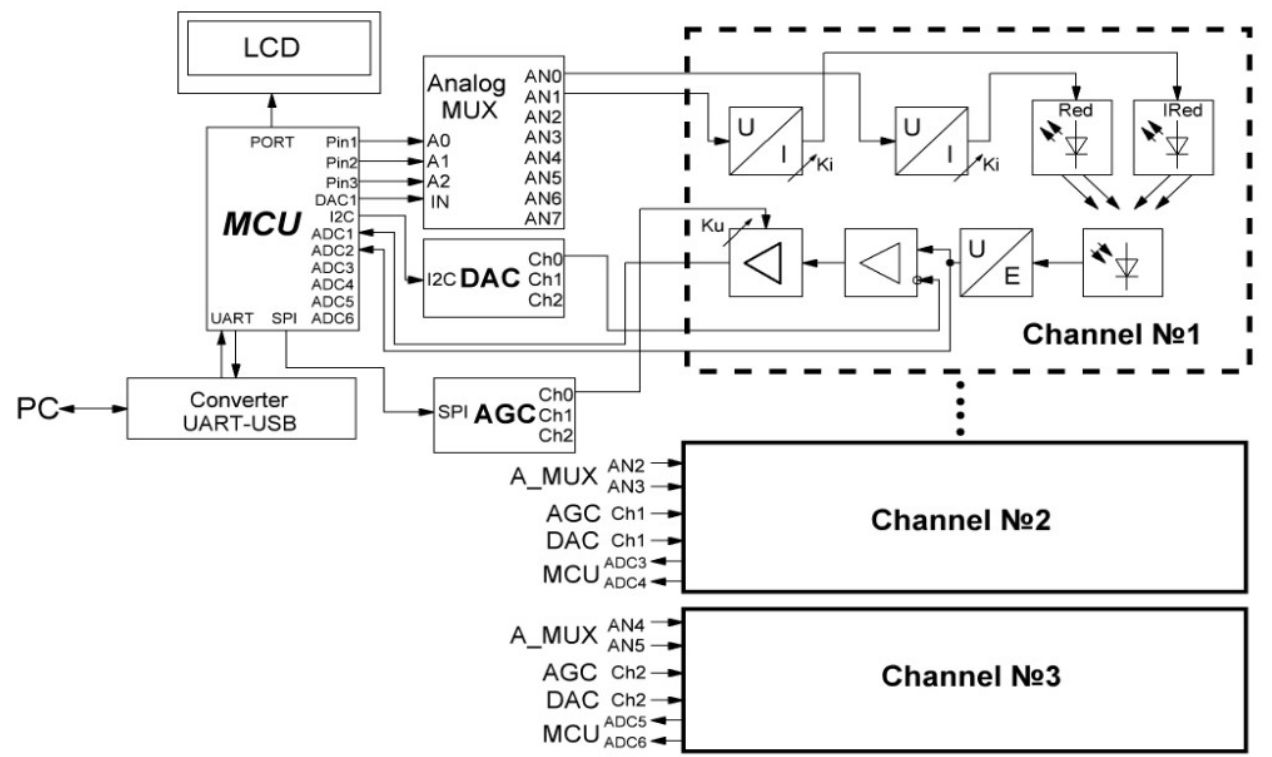

Fig. 2. Schematic diagram of an optoelectronic system for biological tissues investigation. $M C U$ microcontroller unit; $A G C$ - automatic gain control; $D A C$ - digital-to-analog converter; $L C D$ - liquidcrystal display; $P C$ - personal computer; $A D C$ - analog-to- digital converter.

The role of MCU executes the microcontroller STM32F407VG: it organizes the work of all blocks and performs data analysis. Analog multiplexer is used for the intensity modulation of the probing radiation by commutation of the DAC voltage to the voltage-tocurrent converters, which are connected to the laser diodes. The transimpedance amplifiers are used for the signal conversion from the photodiodes. The differential amplifier eliminates an ambient light influence by applying the voltage from the DAC module to the inverting input of the differential amplifier. The applied voltage magnitude equals to the signal value measured using $\mathrm{ADC}$ of microcontroller at the moments when the lasers are turned off. The output stage consists of the inverting amplifier based on the operational amplifier with a digital resistor in the feedback of the amplifier. The digital resistor is used for the realization of the automatic gain control. The AGC unit is an assembly of the digital resistors located in the feedback of the operating amplifier. 


\section{Conclusions}

We developed the schematic diagram of an optoelectronic device intended to eliminate the disadvantages we found in the Infrascanner model 2000. We designed the schematic circuit of an optoelectronic device and created the prototype of the single-channel device. The operation units and device control algorithms will be debugged using the prototype. Currently, the automatic gain control of the output stage has been debugged. The program code for the MCU for coordinated operation of the circuit blocks has been written. The digital filtering of the signal was implemented using a 1st order Butterworth low-pass filter. The connection of the prototype with the PC was completed using UART. The PC application was written for convenient testing of the device.

In the future, for implementation of the triangulation method, the number of channels of the prototype will be increased to three. The device will be tested on the model objects. On the basis of these tests, we plan to develop algorithms, which will allow the detection of hematoma and its parameters, namely: size, depth, and location.

\section{References}

1. The Infrascanner Model 2000, URL: http://infrascanner.com/wpcontent/uploads/2014/12/IFS-Brochure.1.9.15.pdf

2. S. Bressan, M. Daverio, et al., Childs Nerv Syst 30, 477 (2013)

3. C.S. Robertson, E.L. Zager, et al., Journal of Neurotrauma 27, 1597 (2010)

4. A.P. Novoseltseva, K.A. Timchenko, A.A. Aristov, D. Kustov, I.A. Larioshina, Journal of Physics: Conference Series 671, 012002 (2016)

5. K.A. Timchenko, A.A. Aristov, I.S. Musorov, T.G., Evtushenko, Proceedings of the 15th International Conference of Young Specialists on Micro/Nanotechnologies and Electron Devices, 319 (2014)

6. A.P. Novoseltseva, K.A. Timchenko, A.A. Aristov, IOP Conf. Ser: Mat.Sc. and Eng. 93, $012022(2015)$ 